Silvana Nair Leite 1

Maria da Penha Costa Vasconcellos 2

1 Faculdade de Farmácia,

Universidade do Vale

do ItajaÂRua U ruguai 458

Centro, 88302-202,

Itajal SC.

snleite@ccs.univali.br

2 Faculdade de Saúde

Pública/USP

\title{
Adesão à terapêutica medicamentosa: elementos para a discussão de conceitos e pressupostos adotados na literatura*
}

\author{
Adherence to prescribed therapy: \\ points for concepts and presuppositions discussion
}

Abstract Research and discussion on compliance with medical regimens have been taken because compliance is an important part of a treatment. This paper discusses the meanings adopted to compliance and the factors associated to it in the current literature.

Presuppositions, concepts, methodologies, target public and the doctor and patient roles approach of studies on compliance were analyzed. The factors associated with compliance referred in the literature, such as the type of treatment, illness and doctors communication; the qualitative, direct and indirect methodologies adopted, many times with inspect character, were also discussed. We conclude that studies of medications use generally do not emphasize the patient role in the health/illness/treatment process, and so, can not be as comprehensive as necessary.

Key words Compliance, Adherence, Medical regimens, Use of medication, Patient role
* Apoio financeiro da Capes, Univali, Opas - Organização Pan-Americana de Saúde
Resumo A questão da adesão à terapêutica tem sido discutida e estudada por profissionais de saúde por se tratar de um ponto fundamental para a resolubilidade de um tratamento. Neste artigo são discutidos, a partir de estudos de adesão à terapêutica medicamentosa relatados na literatura, os significados adotados para o fenômeno da adesão e os fatores a ele relacionados. Estudos de adesão à terapêutica foram analisados quanto aos seus conceitos e pressupostos, metodologias empregadas e o papel designado para profissionais e pacientes envolvidos. São discutidos os pressupostos teóricos encontrados nos estudos, e os fatores relacionados ao tema, que envolvem questões relativas ao tratamento, ao profissional de saúde e à doença, e as metodologias adotadas nestes estudos, que são baseadas em métodos diretos e indiretos e qualitativos, alguns com abordagem fiscalizadora. Concluise que os estudos no campo dos medicamentos em geral não priorizam o papel do paciente no processo saúde/doença/tratamento, comprometendo a capacidade dos estudos de obter uma compreensão mais ampla e profunda sobre o tema.

Palavras-chave Adesão à terapêutica, Uso de medicamentos, Papel do paciente, Tratamento medicamentoso 


\section{Introdução}

Enquanto $o$ al to consumo de medicamentos é demonstrado e discutido por diversos estudos e desperta preocupação em profissionais e autoridades de saúde, a questão da não-adesão ao tratamento medicamentoso prescrito também tem tomado importância nas últimas décadas e está sendo incluída na lista de preocupações dos profissionais de saúde, juntamente com outros fatores que influem sobre uso racional de recursos terapêuticos. Se há na população o "folclore hipocondríaco", como cita Bucher (1992), por que, então, tantas pessoas não adotam o tratamento que lhes é prescrito ou não 0 realizam adequadamente?

Várias respostas são ensaiadas em estudos de adesão. $E$ a conclusão é que "a não-adesão, em algum grau, é universal" (Jordan et al., 2000) e está relacionada a diversos fatores relativos ao profissional de saúde, ao tratamento, à patologia e ao paciente (Paulo e Zanini, 1997; Nemes, 2000; Vasconcelos, 1996). E apesar de Ruejon (citado por Chaisson, 1998) afirmar que "o normal é não aderir", na população a adesão ao prescrito profissionalmente depende de uma série de fatores, uma espécie de triagem, para que venha a acontecer, enquanto as indicações populares, como as da vizinha ou família, são aceitas mais facilmente (Leite, 2000). Portanto, há o desejo de utilizar al gum tipo de tratamento, de tratar os sintomas e obter alívio, mas há controvérsias quanto à compreensão desse fenômeno.

A relevância da questão na terapêutica é indiscutível: da adesão ao tratamento depende o sucesso da terapia proposta, a cura de uma enfermidade, o controle de uma doença crônica, a prevenção de uma patologia. E se o paciente não adere? Por que isso acontece? Será que o paciente sabe o que é aderir ou tem consciência da questão?

Este artigo tem o intuito de discutir, a partir de uma reflexão teórica da literatura, os significados adotados pelos autores sobre a adesão à terapêutica, os fatores a ela relacionados e a compreensão que se tem sobre o fenômeno. Esta leitura reflexiva de trabal hos sobre 0 assunto pode ajudar a compreender esse universo complexo no qual aparecem diferentes formas de investigação sobre o conceito.

\section{Metodologia}

0 conceito "adesão à ter apêutica" conta com uma vasta literatura, especialmente focando a adesão ao tratamento de uma determinada enfermidade, como nos casos de Aids, tuberculose, hipertensão, ou em determinados grupos populacionais, como crianças e idosos. Selecionar literaturas que proporcionem uma visão do tema de forma geral torna-se uma tarefa complicada. Para a elaboração desta discussão, foram selecionados artigos e livros que tratam do assunto de forma geral e também al guns que tratam da adesão em casos determinados, com o intuito de avaliar os estudos de adesão de diferentes fontes, perspectivas e populações. A consulta a publicações sobre 0 tema na fase de construção de projeto de tese de doutoramento possibilitou constatar a ausência consensual sobre 0 conceito. 0 objetivo do artigo é o de destacar alguns trabalhos que representam diferentes abordagens sobre $o$ tema. Portanto, a literatura aqui mencionada não tem o intuito de ser uma típica revisão de literatura; representa apenas parte dos trabaIhos que colaboraram para a elaboração da discussão. Para tanto, foram selecionados artigos científicos e livros nacionais e internacionais, sem distinção de patologias abordadas, mas os que discutissem o conceito de adesão à terapêutica e que trouxessem abordagens metodológicas diferenciadas. N este campo foram utilizados os textos de literaturas médicas como o estudo de Jones (1983), na Clinical Pediatrics, o de Cramer et al. (1989) publicado pelo jornal da Associação M édica Americana e o de Pessoa et al. (1996) da Revista Paulista de Pediatria; dois textos publicados em uma edição sobre o tema da Controlled Clinical Trials (2000) e o livro de Paulo e Zanine (1997). De revistas da área da saúde geral ou saúde pública foram utilizados os estudos de Brand et al. (1977), de Al-Shammari et al. (1995) e Silvestre-Busto et al. (2001), todos utilizando metodologias quantitativas. Estudando o tema na abordagem da pesquisa qualitativa, foram utilizados os textos de Conrad (1985) publicado pela Social Science and Medicine, de Dowell e Hudson (1997) publicado na Family Practice e de Gonçalves et al. (1999) publicado pelos Cadernos de Saúde Pública. Livros publicados no Brasil recentemente que abordam o tema de forma interdisciplinar, como os de $\mathrm{N}$ emes (2000) e Teixeira et al. ((2000), também são citados. 
Buscando compreender o tema de forma mais ampliada e integrada à problemática saúde/ doença/tratamento, vários textos relacionados ao uso de medicamentos serviram de base para a reflexão: sobre o uso racional de medicamentos e o mercado farmacêutico, lista-se Bucher (1992), Balassubramaniam (1996), Chaisson (1998), Haaijer-Ruskamp eH emminki (1993), Leite (2000), Sobravime (2001); sobre as concepções populares de terapêutica e itinerários terapêuticos foram consultados os estudos de Loyola (1982) em Médicos e curandeiros, Boltanski (1985) em As classes sociais e o corpo, os textos de Menendez (1992) em "Grupo doméstico y proceso saúde/enfermidad/atención" (Cuadernos Médico Sociales), Pachter (1994) em "Culture and clinical care" (JAMA), Vasconcelos (1996) e Ferreira (2001) em "A saúde em comprimidos" (Saúde em Debate).

Além desses, ainda foram utilizadas literaturas sobre metodologia de estudo e métodos de estudos de utilização de medicamentos: Daly et al. (1997), Castro (2000), M ilstein-M oscati et al. (2000).

Foi realizada, inicialmente, a leitura de toda a literatura selecionada. A partir do conhecimento inicial sobre 0 assunto, alguns aspectos foram ressaltados para o aprofundamento da análise e discussão:

- os conceitos de adesão e pressupostos adotados;

- os fatores considerados relativos à adesão; e

- a metodologia utilizada.

Foi útil nessa fase a orientação de Daly et al. (1997) para a análise de textos e materiais gráficos, que sugere ao pesquisador desvendar pontos explícitos e implícitos no material analisado. 0 s autores recomendam questionar 0 material quanto ao tom do texto, a linguagem utilizada, o propósito da história, os termos contraditórios utilizados, se há significados implícitos, se há "heróis e vilões" na história. Dessa forma, a análise do material torna-se aprofundada revelando pontos importantes para a discussão do tema proposto.

\section{Discussão dos temas em estudo}

Conceito de adesão

e pressupostos assumidos

0 conceito de adesão varia entre diversos autores, mas, de forma geral, é compreendido como a utilização dos medicamentos prescritos ou outros procedimentos em pelo menos $80 \%$ de seu total, observando horários, doses, tempo de tratamento. Representa a etapa final do que se sugere como uso racional de medicamentos.

Entre os pressupostos assumidos pelos diversos autores para 0 estudo da adesão, as diferenças mais evidentes encontram-se entre aqueles que focalizam o fenômeno no paciente e aqueles que procuram a compreensão em fatores externos ao paciente.

Os termos utilizados para definir a adesão refletem qual a compreensão que os autores possuem sobre o papel dos atores no processo. Segundo Brawley e Culos-Reed (2000), os termos mais utilizados na língua inglesa adherence e compliance tem significados diferentes. $\mathrm{Pa}$ ra os autores, o termo compliance, que pode ser traduzido como obediência, pressupõe um papel passivo do paciente, e adherence, ou aderência como o termo utilizado para identificar uma escolha livre das pessoas de adotarem ou não certa recomendação. 0 entendimento do papel do paciente como sujeito ativo, que participa e assume responsabilidades sobre seu tratamento, que adere ou não, é defendido por outros autores, como Milstein-M oscati et al. (2000), Conrad (1985) e Dowell e Hudson (1997).

A forma como é visto o papel do paciente no seu tratamento é refletida também na forma como são discutidos os fatores relativos ao paciente na adesão, variando entre a tentativa de compreensão de seus valores e crenças em relação à saúde, à doença e ao tratamento, atéa identificação da não-adesão como comportamento desviante e irracional. Neste último caso, a responsabilidade pela não-adesão ao tratamento é definida como ignorância dos pacientes ou responsáveis por eles sobre a importância do tratamento, a pouca educação da população (presumindo que seria um comportamento típico de classes menos privilegiadas), ou como simples desobediência de "ordens médicas".

Kidd e Altman (2000) citam que nenhum homem é uma ilha, e que essa concepção é verdadeira quando se estuda a questão da adesão. A adesão, como um dos critérios do uso de medicamentos e do cuidado de saúde como um todo, é afetada ou direcionada pelo meio social e cultural em que acontece. A influência social sobre o fenômeno é bastante citada, principalmente estratificando os dados por faixa salarial e escolaridade. Poucos trabal hos, porém, avaliam realmente a questão social e cultural sobre 
o uso de medicamentos como resultado de um processo de decisão racional que leva em conta diversos determinantes, da disponibilidade à crença no tratamento (Haaijer-Ruskamp eH emminki, 1993), resultado da dinâmica de aprendizagem social vivida (Brawley e Culos-Reed 2000) e da forma de construir seu enfrentamento à doença (Dowell e Hudson 1997). Segundo Kidd e Altman (2000), essa construção deve ser compreendida não só no nível individual, mas também no meso e macro contexto, ou seja, nos níveis da comunidade e sociedade como um todo.

\section{O porquê: fatores relacionados à adesão}

A maioria dos estudos de adesão é focada na grande questão que atormenta os profissionais de saúde: por que certos pacientes não aderem ao tratamento medicamentoso?

A forma como é apresentada a questão da adesão pelos autores, a forma como a questão é pensada, elaborada, reflete-se nas categorias de anál ise determinadas para os estudos. Assim, os fatores relacionados ao fenômeno são lançados pelos pesquisadores para verificação nos estudos conforme seus pressupostos sobre o tema, como abordado anteriormente.

Entre os diversos fatores já citados na literatura, pode-se pensar primeiramente na falta de acesso ao medicamento. 0 mercado farmacêutico está concentrado nos países economicamente mais ricos e voltado às classes sociais mais abastadas. Sabe-se que os maiores investimentos estão no desenvolvimento de tratamentos para problemas que atingem essas populações, em detrimento dos medicamentos demandados pelas necessidades mais comuns nos países mais pobres (Sobravime, 2001). Apesar de a despesa com medicamentos representar grande parte do investimento em saúde pública, em países como o Brasil a dispensação gratuita de medicamentos não cobre as necessidades correntes, apesar dos grandes avanços nesse sentido como no caso da terapia anti-retroviral.

No estudo realizado por Brand et al. (1977), o principal fator relatado para a nãoaderência ao tratamento por idosos foi 0 alto custo do medicamento. $E$ as evidências disso estavam nos custos: o custo mensal das prescrições dos pacientes não-aderentes era em média o dobro do custo mensal das prescrições dos aderentes. Logo, pode-se perceber que o maior número de medicamentos prescritos custa mais, e se adere menos. Pessoa et al. (1996) também encontraram o acesso econômico como causa da não-adesão à prescrição após atendimento em pronto-socorro pediátrico em $39,1 \%$ dos casos, eque o menor número demedicamentos e o menor custo melhoram a adesão. Esse fator, sem dúvida, é um grande entrave para a adesão e deve ser o primeiro aspecto a ser avaliado, no entanto al guns estudos que relatam alto índice de não-adesão não referenciam a questão da disponibilização dos medicamentos, demonstrando que, para esses autores, 0 acesso ao medicamento não é um entrave importante. Em países como o Brasil, no entanto, é um problema crucial e deve ser o primeiro fator analisado: se o paciente tem acesso ao medicamento, então ele está em condições para aderir ou não ao tratamento.

0 maior número de medicamentos prescritos e o esquema terapêutico também estão associados à não-adesão mesmo quando o medicamento é fornecido. Esse é um dos principais fatores relacionados ao medicamento que interferem na adesão aos anti-retrovirais, pois os esquemas terapêuticos normalmente são complicados e exigem um grande empenho por parte do paciente, que precisa adaptar sua alimentação, horários e ritmo diário para cumprir o tratamento (Nemes, 2000; Jordan et al., 2000). Silvestre-Busto et al. (2001) encontraram evidências que um tratamento de duas doses diárias é melhor cumprido que de três doses e que, apesar de vários estudos indicarem 0 decréscimo da adesão em tratamentos longos, na antibioticoterapia de crianças em seu estudo esse dado não se confirmou. Além disso, também é importante citar que a percepção de efeitos colaterais causados pela terapia é um entrave para a adesão, o que pode ser chamado de efeito protetor da não-adesão, que seria uma não-adesão inteligente à terapêutica ( $M$ ilsteinM oscati et al., 2000). Vê-se aqui que esses fatores, normalmente identificados ao medicamento, na verdade estão relacionados à decisão do paciente de mudar seu ritmo de vida ou aceitar certos efeitos adversos.

Outro fator citado na literatura éa própria doença. Vários estudos relatam que o tipo de enfermidade tratada parece ter al guma relação com a adesão ou não ao tratamento, o que pode ser lido como a forma como o paciente vê seu estado e compreende sua enfermidade. A ausência de sintomas, em algumas fases do processo de adoecimento, por exemplo, é um dos fatores citados para a não-adesão à terapia da Aids (Teixeira et al., 2000), assim como atra- 
pal ha o seguimento do tratamento da tuberculose (Gonçalves et al., 1999). Alguns estudos de adesão em pediatria citam o fato de os pais não compreenderem a importância do tratamento ou a gravidade da enfermidade como um entrave para a adesão (Pessoa et al., 1996; Jones, 1983), mas também que a percepção de maior gravidade da patologia está associada a maior adesão, mesmo em tratamentos mais longos (Silvestre-Busto et al., 2001).

Certas publicações trazem como questão central a importância do profissional de saúde para a adesão; algumas tratam a questão com importância extrema, como se a adesão ao tratamento fosse determinada exclusivamente pelo poder do médico de fazer seu paciente obedecer a sua prescrição e dos meios que utiliza para tal. De qualquer forma, fica evidente, pelos resultados de diversos estudos, que um dos fatores decisivos para a adesão é a confiança depositada pelo paciente na prescrição, na equipe de saúde ou no médico pessoalmente (Teixeira et al., 2000; Balassubramaniam, 1996; Nemes, 2000; Paulo e Zanini, 1997; Al-Shammari et al., 1995).

Certas atitudes do prescritor, como linguagem, tempo dispensado para a consulta, atendimento acolhedor, respeito com as verbalizações e questionamentos dos pacientes e motivação para o cumprimento da terapia são fatores citados na literatura. Entrevistando pacientes e profissionais de uma unidade de saúde de Itajaí (SC), a questão da linguagem e do background cultural apareceu como um fator extremamente relevante. Os auxiliares de saúde, pessoas da própria comunidade, com baixo grau de especialização, que compartilhavam as mesmas crenças e costumes relativos à saúde da comunidade, conseguiam que a clientela da unidade aderisse às suas recomendações, mesmo entre aqueles pacientes que não costumavam aderir à prescrição dos médicos da unidade. As recomendações e receitas de vizinhos e parentes também eram facilmente atendidas. Dentre os profissionais de saúde, aqueles que usavam linguagem mais popular demonstravam mais respeito pelo paciente e suas crenças, assumiam atitude menos discriminadora e eram mais acreditados pelos clientes da unidade (Leite, 2000). A pesar da nítida influência sobre a adesão, a relação médico-paciente não é suficiente para explicar o fenômeno da não-adesão à terapêutica. É, sim, um dos possíveis entraves que pode e deve ser trabalhado pela equipe de atenção à saúde.
Alguns estudos, entretanto, focalizam no paciente (ou usuário de medicamentos) a questão da adesão. D owell e Hudson (1997), estudando o tema a partir da perspectiva do usuário, descrevem um "modelo de decisão terapêutica". Segundo o modelo, há na população de usuários de medicamentos três tipos: os que aceitam e procuram cumprir a prescrição médica, os que aceitam as prescrições, porém não sem testar variações da prescrição e então optar pelo seguimento ou não, e os sépticos, que não aceitam as prescrições médicas. De acordo com os autores, a aceitação do tratamento está intimamente relacionada com a aceitação da própria doença e não tanto com outros fatores.

Conrad (1985) denomina essa espécie de teste que alguns pacientes mais ativos desenvolvem com seu tratamento de auto-regulação (self-regulation) e o descreve como uma forma de avaliar o estado atual da doença e da possibilidade de retirar ou diminuir a dose de medicamentos, que acontece principalmente em portadores de doenças crônicas. Isso, com a intenção de superar o estigma de doente crônico, pois 0 ato de tomar o medicamento regularmente demonstra, o tempo todo, que se é um doente crônico. Além disso, estudos sobre a adesão a diversas terapias sugerem que o seguimento rígido das prescrições implica certa interferência no cotidiano, o que não é desejado pelo usuário.

Os autores analisados colocam a necessidade de se compreender questões relacionadas ao paciente e ao meio em que está inserido, sociocultural, como importantes no procedimento de adesão à prescrição medicamentosa. Considerando os fatores citados relacionados à doença, ao tratamento e ao serviço de saúde, o que se percebe nos estudos é que mesmo alterando as situações, o grau de adesão continua sendo baixo. Entre os estudos de adesão disponíveis, o grau de adesão varia muito dependendo do método e do conceito de adesão utilizado, ficando em torno de $50 \%$ para a população infantil, por exemplo (Jones, 1983), o que leva a acreditar que os fatores relacionados ao paciente, mais dificilmente controlados, tenham sempre um peso grande na questão da adesão.

É importante destacar que a maioria dos estudos relatados consideram o fenômeno pós consulta médica e avaliam, em geral, o cumprimento da prescrição médica como cena principal do fenômeno saúde/doença/atenção. Porém o fenômeno da adesão-não-adesão pode ser compreendido desde a escolha do sistema 
terapêutico: todas as motivações e bloqueios estarão presentes em todos os níveis da terapêutica, afetando a relação do paciente ou população com sua saúde e terapêutica. Todos os fatores apontados para a adesão a um tratamento prescrito estão influenciando o modo pelo qual uma pessoa, na ocorrência de uma enfermidade, decidirá tratar-se.

\section{Considerações sobre os métodos para o estudo da adesão}

Quando o foco do estudo é adesão ao tratamento prescrito, os métodos podem ser classificados em diretos e indiretos, sendo que não há um método considerado gold standard, como cita Cramer et al. (1989). Os métodos diretos são fundamentados nos ensinamentos de Hipócrates, que já recomendava desconfiar das informações fornecidas pelos pacientes em relação ao tratamento. São baseados em técnicas analíticas que verificam se o medicamento foi administrado ou tomado na dose e freqüência necessária através da identificação de metabólitos do medicamento ou de marcadores químicos de maior permanência no organismo ( $M$ ilstein-M oscati et al., 2000). Os métodos indiretos incluem entrevistas e a contagem das unidades de medicamentos que o paciente ainda possui. Por desconfiar da fidedignidade do método, pois o paciente pode eliminar as doses que não foram utilizadas, alguns estudos utilizam um dispositivo para acondicionar o medicamento que registra os intervalos de retirada das doses, porém mesmo assim pesquisadores como Cramer et al. (1989) cogitam a possibilidade de 0 paciente retirar a dose da embalagem e não tomá-la. A própria avaliação do efeito farmacológico esperado é um método para avaliar a adesão ao tratamento, ficando difícil nesse caso avaliar se a resposta terapêutica é diretamente proporcional ao tratamento prescrito.

Um dos métodos mais utilizados é a entrevista estruturada, por sua aplicação mais acessível e de menor custo. 0 maior problema apontado para esse método éa superestimativa da adesão, pois mais uma vez o paciente pode esconder do entrevistador ou do médico a forma como realizou o tratamento na realidade (M ilstein-M oscati et al., 2000).

A limitação dos questionários estruturados, com questões objetivas, é discutida para qualquer tipo de levantamento. Questões como 0 constrangimento do entrevistado, seu desejo de responder 0 que seria o correto e a pressão, mesmo que não intencional, da inquisição fazem deste um método superficial. Todas essas considerações acerca da fidedignidade dos mé todos são reflexos do tipo de abordagem adotado nos estudos, que não prevê nenhum grau de aproximação com a realidade do paciente e de seu meio e, portanto, não inspira relação de confiança entre paciente e entrevistador, e nem compreensão do conjunto de signos que permeiam a questão do uso de medicamentos.

Em nenhum momento écitado o direito do paciente de decidir sobre o seu corpo. Criamse engenhocas eletrônicas e formas de cercar 0 paciente por todos os lados e este necessita prestar contas do que faz e deixa de fazer com seu próprio corpo. Alguém já se perguntou com que direito o pesquisador pode colocar pacientes nessa situação de inquisição? N ão se pretende, com esta questão, desprezar os dados de estudos tradicionais de adesão à terapêutica, mas alertar para os significados que dados obtidos nestes modelos podem representar.

H aaijer-Ruskamp e Hemminki (1993), quando citam a pesquisa qualitativa na área de medicamentos de forma geral, referem-se aos mé todos chamados qualitativos, como a pesquisa antropológica. N esse caso, são desenvolvidas metodologias que propiciam o estudo de determinados fenômenos em profundidade, como a pesquisa participante, a etnografia e as entrevistas abertas, focando os objetivos do estudo em fatores sociais, culturais e psicológicos e sua influência no uso de medicamentos, como alguns ensaios sobre adesão à terapia anti-retroviral (Nemes, 2000; Teixeira et al., 2000). Esse tipo de abordagem pode contemplar a compreensão dos significados do uso de medicamentos e utilizar a contribuição das ciências sociais para o entendimento da questão, principalmente porque consideram o campo da saúde um corpo complexo de conhecimentos que vão nortear as atitudes em relação aos cuidados de saúde, entre eles, e não à margem deles, a utilização de medicamentos. A perspectiva do usuário é uma das maiores contribuições da abordagem, propiciando a emergência de fatores complexos até então desconsiderados, como nos estudos de Dowell e Hudson (1997) e Conrad (1985), que identificaram a adesão à terapêutica prescrita como o resultado de um processo de avaliação e aceitação de seu estado enquanto doente.

Alguns formulários para entrevistas direcionadas para adesão são descritos na literatura, como o teste de Haynes-Sacket e o de M o- 
risky, Green e Levine (1986). No primeiro caso, o questionamento sobre a adesão ou não à terapêutica é feito de forma amigável, tentando expressar o menor nível de pressão possível, citando as dificuldades en contradas por outros pacientes, e solicitando que o paciente fale sobre suas dificuldades. 0 segundo é o mais usado e validado em espanhol para pacientes asmáticos e hipertensos (Silvestre-Busto et al., 2001). Constituído de quatro perguntas básicas sobre horário, esquecimento, percepção de efeitos colaterais e a ausência de sintomas, 0 teste tende a superestimar a não-adesão, ao contrário do que se espera de métodos indiretos, e não dá ao paciente a oportunidade de expressar suas dificuldades e seu entendimento a respeito do tratamento. A pesar dos testes serem baseados em formulários padronizados, sem aprofundamento individualizado, al guns autores citam a necessidade de que o paciente e entrevistador tenham uma relação de confiança e de que a validade dos testes depende da sinceridade do entrevistado.

\section{Reflexões finais}

0 tema deste artigo, adesão à terapêutica medicamentosa, merece uma profunda e continuada reflexão sobre seus significados e "ressignificados" nos atos de prescrição e dispensação e na forma de consumo, por parte do profissional de saúde e do paciente. A análise de estudos de adesão aqui relatada levanta algumas questões que merecem ser discutidas. Uma delas éa questão do papel do paciente no processo saúde/doença/tratamento. M uitos estudos no campo dos medicamentos ainda desconsideram o paciente, ou o responsável por sua saúde, como ser social, dotado de expectativas, conhecimentos, interesses, e que tem valores socioculturais, que produzem sentidose "ressignificações" sobre o uso ou resistência ao uso medicamentoso em seu processo ou estado crônico de adoecimento. 0 paciente é referido como um espaço, o lócus onde um medicamento terá sua ação do ponto de vista da racionalidade médica (Luz, 1988).

Podemos observar a partir de leitura mais aprofundada das questões, excetuando o qua- dro em que o paciente não tem acesso ao medicamento, que o número de doses ou os efeitos adversos serão empecilho para a adesão dependendo da percepção que o paciente tem disso; que a própria doença é um fator interferente dependendo da forma como o paciente enfrenta a doença e seus sintomas, e que o profissional de saúde influenciará na adesão na medida em que atingir o universo cultural do paciente e estabelecer com este comunicação e relacionamento efetivos, incluindo valorização do consumo como benefício em seu corrente cotidiano, quando este se fizer necessário.

Portanto, é o paciente, ou o responsável por ele, o objetivo e o objeto das investigações e das ações para promover a adesão. N ele estão centrados os fatores que interferem na adesão à terapêutica, refletindo o contexto individual, familiar e social. No entanto, a questão da adesão é discutida no nível profissional e reflete as expectativas e opiniões profissionais. Motivos considerados sem importância pelo profissional de saúde muitas vezes são aqueles que realmente determinam o seguimento ou não de determinado tratamento pelo paciente (Teixeira et al., 2000). Chama especial atenção a conclusão de Al-Shammari et al. (1995) em seu estudo, em que a maioria dos pacientes com ausência de adesão à terapêutica prescrita mencionou apenas poucos motivos para isto.

Como poderiam relatar seus motivos? Apesar de haver em uma parcela da população a consciência sobre seu tratamento e deste acontecer como resultado de um processo de decisão (Dowell e Hudson, 1997), ele não está mais preocupado com o seguimento do tratamento propriamente dito do que com a mel hora dos sintomas, a volta à vida normal, a superação da fragilidade. Isso Ihe garantirá segurança e alívio e não importa o meio, se um medicamento, uma reza ou simplesmente "esquecer" que 0 problema existe. Como explicita Conrad (1985), o paciente não está preocupado em desobedecer ou não aderir ao receituário médico, mas sim em lidar com sua condição de vida da forma que Ihe convenha e que Ihe permita mai or autocontrole eliberdade. 


\section{Referências bibliográficas}

Al-Shammari SA, Khoja T, Al-Yamani MJM S 1995. Compliance with short-term antibiotic therapy among patients attending primary health centers in Riyadh, Saudi Arabia. Journal of Royal Society of Health, Aug: 231-234.

Balassubramaniam K 1996. Rational use of drugs in children, pp. 19-190. In The rational use of drugs. Zhulian, Penang.

Boltanski LUC 1985. As classes sociais e o corpo. Graal Editora, São Paulo.

Brand FN, Smith RT \& Brand PA 1977. Effect of economic barriers to medical care on patient's noncompliance. Public Health Reports 92:72-78.

Brawley LR \& Culos-Reed N 2000. Studying adherence to therapeutic regimens: overview, theories, recommendations. Controlled Clinical Trials 21:156s-163s.

Bucher R 1992. Drogas e drogadição no Brasil. Editora Artes M édicas, São Paulo.

Castro CGSO (org.) 2000. Estudos de utilização de medicamentos: noções básicas. Fiocruz, Rio de Janeiro.

Chaisson R 1998. Manuseio de infecções oportunistas. II Brazil Johns Hopkins University Conference on HIV/AIDS. Disponível em <www.hopkins-aids.edu>

Conrad P 1985. The meaning of medication: another look at compliance. Social Science and Medicine 20 (1):29-37.

Cramer, JA M attson RH, Prevey M L, Séller RD \& Ouellette VL 1989. How often is medication taken as pre scribed? JAMA 261(22):3.273-3.277.

Daly J, Kellehear A \& Gliksman M 1997. The public health researcher: a methodological guide. Oxford University Press, Melbourne.

Dowell J \& Hudson H 1997. A qualitative study of medication-taking behaviour in primary care. Family Practice 14(5):369-375.

Ferreira J 2001. A saúde em comprimidos: influências socioculturais na interpretação de sintomas e terapias medicamentosas em uma vila de classe popular de Porto Alegre. Saúde em Debate 25(59):67-72.

Gonçalves H, Costa JSD, M enezes AM B, Knauth D \& Leal OF 1999. Adesão à terapêutica da tuberculose em Pelotas, Rio Grande do Sul: na perspectiva do paciente. Cadernos de Saúde Pública 15(4):777-787.

Haaijer-Ruskamp FM \& Hemminki E 1993. The social aspects of drug use. In M NG Dukes. Drug utilization studies. WHO Regional Office for Europe, Copenhagen.

Jones JG 1983. Compliance with pediatric therapy. Clinical Pediatrics, apr: 2.262-2.265.
Jordan MS et al. 2000. Aderência ao tratamento antiretroviral em Aids: revisão da literatura médica. In PR Teixeira et al. Tá difícil de engolir? Editora $\mathrm{Ne}$ paids, São Paulo.

Kidd KE \& Altman DG 2000. Adherence in social context. Controlled Clinical Trials 21:184S-187S.

Leite SN 2000. Além da medicação: a contribuição da fitoterapia para a saúde pública. Dissertação de mestrado, Faculdade de Saúde Pública, Universidade de São Paulo, São Paulo.

Loyola M A 1982. Médicos curandeiros. Difel Editora, Rio de Janeiro.

Luz M 1988. Natural, racional, social: razão médica e racionalidade científica moderna. Editora Campus, Rio de Janeiro.

M enendez EL 1992. Grupo doméstico y proceso salud/ enfermedad/atención. Del "teoricismo" al movimiento continuo. Cuadernos Médicos Sociales 59:3-18.

M ilstein-M oscati I, Persano S \& Castro LLC 2000. Aspectos metodológicos e comportamentais da adesão à terapêutica, pp. 171-179. In LLC Castro (org.). Fundamentos de farmacoepidemiologia. AG Editora, [s.I.].

Morisky DE, Green LW \& Levine DM 1986. Concurrent and predictive validity of self-reported measure of medication adherence. Medical Care 24:67-74.

Nemes MIB et al. 2000. Aderência ao tratamento por antiretrovirais em serviços públicos no Estado de São Paulo. Ministério da Saúde, Brasília.

Pachter LM 1994. Culture and clinical care. Journal of American Medical Association 271(9):690-694.

Paulo LG \& Zanini AC 1997. Compliance: sobre o encontro paciente/médico. IPEX Editora, São Roque.

Pessoa JHL, Balikjan P, Frittella S, Nascimento R \& Ribeiro L 1996. Não-adesão à prescrição após atendimento em pronto-socorro pediátrico. Revista Paulista de Pediatria 14(02):73-77.

Silvestre-Busto $C$ et al. 2001. Multi-centre study of childrens's adherence to antibiotic treatment in primary care. Atención Primaria 27(8):554-558.

Sobravime 2001. O que é o uso racional de medicamentos? Sobravime, São Paulo.

Teixeira PR, Paiva V \& Shimma E 2000. Tá difícil de engolir? Editora Nepaids, São Paulo.

Vasconcelos EM 1996. A terapêutica médica e as práticas populares de saúde. Saúde em Debate 49(50):100106.

Artigo apresentado em 22/3/2003

Aprovado em 1ㅇ/4/2003

Versão final apresentada em 12/7/2003 